\title{
Pion condensation in the two-flavor chiral quark model at finite baryochemical potential
}

\author{
T. Herpay $*$ and P. Kováçđ \\ Research Institute for Particle and Nuclear Physics of the Hungarian Academy of Sciences, H-1525 Budapest, Hungary and \\ Research Group for Statistical and Biological Physics of the Hungarian Academy of Sciences, H-1117 Budapest, Hungary
}

\begin{abstract}
Pion condensation is studied at one-loop level and nonzero baryochemical potential in the framework of two flavor constituent quark model using the one-loop level optimized perturbation theory for the resummation of the perturbative series. A Landau type of analysis is presented for the investigation of the phase boundary between the pion condensed/non-condensed phases. The statement that the condensation starts at $\mu_{\mathrm{I}}=m_{\pi}$ is slightly modified by one-loop corrections. The second order critical surface is determined and analysed in the $\mu_{\mathrm{I}}-\mu_{\mathrm{B}}-T$ space. The $\mu_{\mathrm{I}}$ dependence of the one-loop level charged pion pole masses is also studied.
\end{abstract}

PACS numbers: 11.10.Wx, 03.75.Nt, 11.10.6h, 21.65.+f

\section{INTRODUCTION}

From the early 70's, when the idea of pion condensation was first suggested [1], many investigations have been performed in the field. Right in the early works, the possibility emerged that pion condensation might have a significance in the evolution of compact stars [2, 3, 4] . Beside compact stars, pion condensation can occur in asymmetric nuclear matter as well as in heavy ion collisions at intermediate energies. While the phases of QCD can be well described by perturbation theory at extreme high temperatures and densities, at moderate temperatures and densities a perturbative description is not possible, here other methods like lattice field theory and effective field theories can be applied. In lattice field theory a second order phase transition was found from normal phase to pion condensed phase in two flavor QCD at a critical isospin chemical potential, which is about the pion mass [5, 6, 7]. Pion and in some cases kaon condensation was also investigated in numerous effective models such as chiral perturbation theory [8, 9], ladder QCD [10], random matrix method [11], NJL model (in the mean field approximation) [12, 13, 14, 15], PNJL model [16] and linear sigma model [17, 18, 19]. These investigations mostly focus on properties of asymmetric hadronic matter, however if one wants to describe for instance a neutron star, which is electrically neutral on average, then the condition of charge neutrality as well as $\beta$-equilibrium must be imposed explicitly as discussed in [14, 20].

Our main goal here is to go beyond the present status of the field and determine the phase boundary between the pion condensed and non-condensed phases in the $\mu_{\mathrm{I}}-\mu_{\mathrm{B}}-T$ space at one-loop level and to calculate the $\mu_{\mathrm{I}}$ dependence of the charged pion masses beyond tree level. This work is the continuation of our previous works [21, 22], in the sense that we use the same framework of constituent quark model and the optimized perturbation theory (OPT) [23] as a resummation technique, which preserves the perturbative renormalizability as well as the symmetries of the model. However there we used three flavor model, while the present investigation deals with an effective model of 2-flavor QCD. This approximation is sufficient to see the main properties of pion condensation.

The paper is organized as follows. In Section II, we introduce the model and discuss its renormalization in detail in case when not only scalar but also pseudoscalar (pion) condensate is present. We treat bosons at one-loop level, while the constituent quarks are considered at tree level. In Section III, we present the one-loop equations, which describe our system, namely the equations of state for the scalar and pion condensates and the equation for the resummed pion mass. Moreover we show that a Ward identity holds, from which the Goldstone theorem also follows. Then we expand our equations in powers of the pion condensate, with which we restrict ourselves to a Landau-type analysis of the phase transition. At the end of this section a simple formula for the boundary of the pion condensation domain is established. In Section IV we diagonalize the mixed boson and fermion propagators, which are determined also for small values of the pion condensate. Section V is dedicated to the parameterization of the model, i.e. we fix the parameters of the Lagrangian at zero temperature and vanishing chemical potentials at one-loop level. Here we also discuss the choice of the renormalization scales. In Section VI we present the numerical results and finally we conclude in Section VII.

\footnotetext{
*Electronic address: herpay@cleopatra.elte.hu

${ }^{\dagger}$ Electronic address: kpeti@cleopatra.elte.hu
} 


\section{THE MODEL AND ITS RENORMALIZATION}

Our starting point is the renormalized $S U(2)_{L} \times S U(2)_{R}$ symmetric Lagrangian with explicit symmetry breaking term, which contains meson as well as quark fields [24]. Without the symmetry breaking term the symmetry group of the light meson sector $(\pi, \sigma)$ is $O(4)$, and the Lagrangian is

$$
\begin{aligned}
\mathcal{L} & =\frac{1}{2}\left(\partial_{\mu} \phi \partial^{\mu} \phi-m^{2} \phi^{2}\right)-\frac{\lambda}{4} \phi^{4}+h \phi_{0}+\mathrm{i} \bar{\psi} \gamma_{\mu} \partial^{\mu} \psi-\frac{g_{F}}{2} \bar{\psi} T_{\alpha} \phi_{\alpha} \psi \\
& +\frac{1}{2}\left(\delta Z \partial_{\mu} \phi \partial^{\mu} \phi-\delta m^{2} \phi^{2}\right)-\frac{\delta \lambda}{4} \phi^{4},
\end{aligned}
$$

where $\psi=(u, d)^{T}$ are the $S U(2)$ doublet quark fields, $\phi=\left(\phi_{0}, \phi_{1}, \phi_{2}, \phi_{3}\right) \equiv\left(\sigma, \pi_{1}, \pi_{2}, \pi_{3}\right)$ are the scalar sigma and pseudoscalar pion fields, $h$ is the symmetry breaking external field, and $T_{\alpha}=\left(\tau_{0}, \mathrm{i} \tau \gamma_{5}\right), \quad \alpha=0 \ldots 3$ are the quark-boson coupling matrices. In the Lagrangian above the couplings $m^{2}, \lambda$ and the $\phi$ field are renormalized (finite) quantities, while $\delta m^{2}, \delta \lambda$, and $\delta Z$ are the corresponding (infinite) counterterms. Since we treat quarks only at tree level no wave function renormalization constant is necessary for the fermionic fields.

We use the grand canonical generating functional of the renormalized Lagrangian in order to introduce the baryon and isospin chemical potentials as well as the scalar and pion condensates. As one shall see, one consequence of the presence of pion condensation amounts to the appearance of the non-trivial wave function renormalization constant in the equation of state of the condensate. At finite temperature and densities the grand canonical generating functional, which determines the $N$-point functions of the fields, is

$$
\mathcal{Z}=\int \mathcal{D} \phi \mathcal{D} \Pi \mathcal{D} \bar{\psi} \mathcal{D} \psi \exp \left[\mathrm{i} \int_{0}^{-\mathrm{i} \beta} d t \int d^{3} x\left(\Pi \dot{\phi}+\mathrm{i} \bar{\psi} \gamma_{0} \dot{\psi}-\mathcal{H}+\mu_{\mathrm{B}} Q_{\mathrm{B}}+\mu_{\mathrm{I}} Q_{\mathrm{I}}\right)\right],
$$

where the Hamiltonian of the system is

$$
\begin{aligned}
\mathcal{H} & =\frac{1}{2}\left(\Pi^{2}+(\nabla \phi)^{2}+m^{2} \phi^{2}\right)+\frac{\lambda}{4} \phi^{4}-h \phi_{0}+\mathrm{i} \bar{\psi} \gamma_{i} \partial_{i} \psi+\frac{g_{F}}{2} \bar{\psi} T_{\alpha} \phi_{\alpha} \psi \\
& -\frac{1}{2} \delta Z \Pi^{2}+\frac{1}{2} \delta Z(\nabla \phi)^{2}+\frac{\delta \lambda}{4} \phi^{4}+\frac{1}{2} \delta m^{2} \phi^{2}, \quad(i=1,2,3)
\end{aligned}
$$

and the canonical momenta of the scalar fields are defined by

$$
\Pi=\frac{\delta \mathcal{L}}{\delta \dot{\phi}}=(1+\delta Z) \dot{\phi}
$$

In (2) $Q_{B}, Q_{I}$ are the conserved baryon and isospin charges defined by the zeroth component of the corresponding Noether currents of the symmetric Lagrangian

$$
\begin{aligned}
Q_{B} & =\int \mathrm{d}^{3} x \frac{1}{3}\left(u^{\dagger} u+d^{\dagger} d\right), \\
Q_{I} & =\int \mathrm{d}^{3} x\left[(1+\delta Z)\left(\pi_{1} \dot{\pi}_{2}-\pi_{2} \dot{\pi}_{1}\right)+\frac{1}{2}\left(u^{\dagger} u-d^{\dagger} d\right)\right] .
\end{aligned}
$$

At small temperatures, when $h \neq 0$ or $h=0$ and $m^{2}<0$ the original $O(4)$ symmetry of the meson sector breaks down to $O(3)$, which means that the $\sigma$ scalar field has a nonzero vacuum expectation value (vev), thus $\left\langle\phi_{0}\right\rangle \equiv\langle\sigma\rangle \equiv v \neq 0$. Moreover, if the isospin chemical potential $\mu_{\mathrm{I}}$ is sufficiently large, charged pions condensate (Bose-condensation) and another nonzero vacuum expectation value occurs, which additionally breaks down the symmetry to $O(2)$. If one initially introduces vev to all the four bosonic fields it can be shown that because of the remaining $O(2)$ symmetry the fields can be transformed in such a way that only two nonzero expectation value remains, from which one is the aforementioned $v$, and the other one can be either $\left\langle\phi_{1}\right\rangle$ or $\left\langle\phi_{2}\right\rangle$, i.e. one can entirely transform out $\left\langle\phi_{3}\right\rangle$. Hence, we can choose $\left\langle\phi_{1}\right\rangle \equiv\left\langle\pi_{1}\right\rangle \equiv \rho \neq 0$ and $\left\langle\phi_{i}\right\rangle \equiv\left\langle\pi_{i}\right\rangle=0$ for $i=2,3$ (see for e.g. [18]).

Shifting the fields by their expectation values in Eq.(2) the generating functional can be written as

$$
\mathcal{Z}=\int \mathcal{D} \phi \mathcal{D} \bar{\psi} \mathcal{D} \psi\left[\mathrm{e}^{-\int_{0}^{-\mathrm{i} \beta} d t \int d^{3} x \bar{\psi} G^{f-1} \psi} \mathrm{e}^{-\mathrm{i} \int_{0}^{-\mathrm{i} \beta} d t \int d^{3} x \tilde{\mathcal{L}}_{I}} \int \mathcal{D} \Pi \mathrm{e}^{i \int_{0}^{-\mathrm{i} \beta} d t \int d^{3} x\left(\Pi \dot{\phi}-\tilde{\mathcal{H}}_{B}\right)}\right] .
$$

Here $G^{f^{-1}}$ is the tree level fermion propagator matrix, which after going to Fourier space and introducing the $\omega_{n}=(2 n+1) \beta$ fermionic Matsubara frequencies, is given as (see for e.g. [17]),

$$
\mathrm{i} G_{i j}^{f-1}=\left(\begin{array}{cc}
\left(-\mathrm{i} \omega_{n}+\frac{1}{3} \mu_{\mathrm{B}}+\frac{1}{2} \mu_{\mathrm{I}}\right) \gamma_{0}-\gamma_{i} p_{i}-\frac{g_{F}}{2} v & -\mathrm{i} \frac{g_{F}}{2} \gamma_{5} \rho \\
-\mathrm{i} \frac{g_{F}}{2} \gamma_{5} \rho & \left(-\mathrm{i} \omega_{n}+\frac{1}{3} \mu_{\mathrm{B}}-\frac{1}{2} \mu_{\mathrm{I}}\right) \gamma_{0}-\gamma_{i} p_{i}-\frac{g_{F}}{2} v
\end{array}\right)
$$


where $p_{i}$ is the spatial momentum. In (7) $\tilde{\mathcal{H}}_{B}=\tilde{\mathcal{H}}_{B}^{\Pi}+\tilde{\mathcal{H}}_{B, 2}$ contains all terms depending on the canonical momenta and bosonic fields up to second order. Other terms are contained in the $\tilde{\mathcal{L}}_{I}$. The non-zero condensates generate three point couplings, which can be directly read off from the explicit form of $\tilde{\mathcal{L}}_{I}$ A.1. The combination $\Pi \dot{\phi}-\tilde{\mathcal{H}}_{B}^{\Pi}$ can be written as

$$
\begin{aligned}
\Pi \dot{\phi}-\tilde{\mathcal{H}}_{B}= & -\frac{1}{2}(1-\delta Z)\left(\Pi_{0}^{2}-2 \Pi_{0}(1+\delta Z) \dot{\phi}_{0}\right) \\
& -\frac{1}{2}(1-\delta Z)\left(\Pi_{3}^{2}-2 \Pi_{3}(1+\delta Z) \dot{\phi}_{3}\right) \\
& -\frac{1}{2}(1-\delta Z)\left(\Pi_{1}^{2}-2 \Pi_{1}(1+\delta Z)\left(\dot{\phi}_{1}-\mu_{\mathrm{I}} \phi_{2}\right)\right) \\
& -\frac{1}{2}(1-\delta Z)\left(\Pi_{2}^{2}-2 \Pi_{2}(1+\delta Z)\left(\dot{\phi}_{2}+\mu_{\mathrm{I}}\left(\phi_{1}+\rho\right)\right)\right) .
\end{aligned}
$$

After completing into whole squares and performing the integration over the canonical momenta in (9), one can identify the tree level bosonic inverse propagator matrix and from the linear terms the tree level equations of states $(\mathrm{EoS})$.

The $4 \times 4$ inverse boson propagator $\left(G^{b^{-1}}\right)$ in Fourier space with the introduction of the $\omega_{n}=2 \pi n \beta$ bosonic Matsubara frequencies splits up into the inverse propagator of $\pi_{3}$ and a $3 \times 3$ coupled inverse propagator matrix of the remaining three bosonic fields $\pi_{1}, \pi_{2}, \sigma$. In the $\pi_{1,2}$ sector we switch to the charged $\pi^{ \pm}=\left(\pi_{1} \mp \mathrm{i} \pi_{2}\right) / \sqrt{2}$ base and use the following relabeling $i, j=1,2,3,4 \longrightarrow \pi^{+}, \pi^{-}, \sigma, \pi_{3}$. In this way the non zero elements of $G_{i j}^{b-1}$ are

$$
\begin{aligned}
\mathrm{i} G_{44}^{b}{ }^{-1} & =\left(-\mathrm{i} \omega_{n}\right)^{2}-E_{\pi_{3}}^{2}, \\
\mathrm{i} G_{k l}^{b}{ }^{-1}= & \left(\begin{array}{ccc}
\left(-\mathrm{i} \omega_{n}-\mu_{\mathrm{I}}\right)^{2}-E_{\pi_{3}}^{2}-\lambda \rho^{2} & -\lambda \rho^{2} & -\sqrt{2} \lambda v \rho \\
-\lambda \rho^{2} & \left(-\mathrm{i} \omega_{n}+\mu_{\mathrm{I}}\right)^{2}-E_{\pi_{3}}^{2}-\lambda \rho^{2} & -\sqrt{2} \lambda v \rho \\
-\sqrt{2} \lambda v \rho & -\sqrt{2} \lambda v \rho & \left(-\mathrm{i} \omega_{n}\right)^{2}-E_{\pi_{3}}^{2}-2 \lambda v^{2}
\end{array}\right),
\end{aligned}
$$

where $k, l=1,2,3, E_{\pi_{3}}=\sqrt{\mathbf{p}^{2}+m_{\pi_{3}}^{2}}$, and the tree level $\pi_{3}$ mass square is $m_{\pi_{3}}^{2}=m^{2}+\lambda\left(v^{2}+\rho^{2}\right)$.

As mentioned earlier in (9) the coefficients of the terms linear in the corresponding fields $\phi_{0}(\sigma)$ and $\phi_{1}\left(\pi_{1}\right)$ determine the two non-trivial equations of states:

$$
\begin{aligned}
& \operatorname{EoS}_{\sigma}^{\text {tree }}=v\left(m^{2}+\lambda\left(v^{2}+\rho^{2}\right)\right)-h=0 \\
& \operatorname{EoS}_{\pi_{1}}^{\text {tree }}=\rho\left(m^{2}+\lambda\left(v^{2}+\rho^{2}\right)-\mu_{\mathrm{I}}^{2}(1+\delta Z)\right)=0 .
\end{aligned}
$$

In the second EoS the wave function renormalization constant $\delta Z$ is explicitly written - which unavoidably occurs when field renormalization is introduced followed by the canonical way of introducing chemical potentials- even if it is a higher order term, because we would like to emphasize the importance of its presence. From the one-loop fermionic contribution of the second EoS, as it will be seen later, a $\mu_{\mathrm{I}}^{2}$ proportional divergence arise, which has to be absorbed into some infinite counterterm, and this counterterm will be the $\delta Z$ renormalization constant. In other words the lack of $\delta Z$ would result in an uncanceled divergence in the second EoS.

The infinite parts of the counterterms can be obtained by requiring the finiteness of the perturbative $N$-point functions (in our case the propagator and the four point boson vertex) in the symmetric phase $(v=\rho=0)$ at $T=\mu_{\mathrm{B}}=\mu_{\mathrm{I}}=0$. Practically it is easier to obtain the infinite parts from the one-loop EoS (see Sec. III). Accordingly, the following counterterms can be found up to one-loop order with cutoff regularization,

$$
\begin{aligned}
\delta m^{2} & =-6 \lambda\left(\Lambda^{2}-m^{2} \ln \frac{\Lambda^{2}}{l_{b}^{2}}\right)+\frac{g_{F}^{2}}{4 \pi^{2}} N_{c} \Lambda^{2}, \\
\delta \lambda & =12 \lambda^{2} \ln \frac{\Lambda^{2}}{l_{b}^{2}}-\frac{g_{F}^{2}}{32 \pi^{2}} N_{c} \ln \frac{\Lambda^{2}}{\mathrm{e}_{f}^{2}}, \\
\delta Z & =-N_{c} \frac{g_{F}^{2}}{16 \pi^{2}} \ln \frac{\Lambda^{2}}{l_{f}^{2} \mathrm{e}^{2}},
\end{aligned}
$$

where $\Lambda$ is the three dimensional cutoff in momentum space, while $l_{b}$ and $l_{f}$ are the bosonic and fermionic renormalization scales, respectively. These counterterms cancel by definition all divergences at one-loop level and at $v=\rho=T=\mu_{\mathrm{B}}=\mu_{\mathrm{I}}=0$ and in the broken phase the same counterterms can be used with a slight modification due to the necessary resummation (see Sec. III). The finite parts of $\delta m^{2}, \delta \lambda$ and $\delta Z$ are determined by the parameterization of the model in the broken phase (see Sec. IV). 


\section{EQUATIONS AT ONE-LOOP LEVEL}

As it is well known from finite temperature field theory, tree level mass squares can become negative in the broken phase as the temperature increases. Accordingly some sort of resummation of the perturbative propagator is needed (see for example in [25]). We perform this using the optimized perturbation theory [23], where a temperature (and chemical potential) dependent mass term is introduced in the Lagrangian and the difference between the original and the new mass parameter is treated as a (finite) higher order counterterm,

$$
\mathcal{L}_{\text {mass }}=-\frac{1}{2} m^{2} \phi^{2}=-\frac{1}{2} M^{2}(T, \mu) \phi^{2}-\frac{1}{2} \Delta m^{2}(T, \mu) \phi^{2} .
$$

Here $\Delta m^{2}$ is the finite "one-loop level" counterterm. The new mass parameter is determined by requiring that the inverse one-loop level $\pi_{3}$ propagator at zero external momentum stays equal to its tree level value (fastest apparent convergence FAC)

$$
M_{\pi_{3}}^{2} \equiv M^{2}+\delta m^{2}+(\lambda+\delta \lambda)\left(v^{2}+\rho^{2}\right)+\Sigma_{\pi_{3}}\left(\omega=\mathbf{p}=0, M^{2}, T, \mu\right)+\Delta m^{2}=M^{2}+\lambda\left(v^{2}+\rho^{2}\right) \equiv m_{\pi_{3}}^{2},
$$

where we indicated the resummed mass dependence of the self energy $\left(\Sigma_{\pi_{3}}\right)$. Strictly speaking the OPT at one-loop order replaces $m$ with $M(T, \mu)$ also in the internal tree level propagator lines. Since $\pi_{3}$ is not a mixed state, $M(T, \mu)$ can be expressed through the tree level $\pi_{3}$ mass, which equals its one-loop level value by the condition (15), and thus the diagonal part of the boson propagator (10) can be written as a function of the $\pi_{3} \operatorname{mass}$ instead of $M(T, \mu)$,

$$
\begin{aligned}
& \mathrm{i} G_{11}^{b}{ }^{-1}=\left(-\mathrm{i} \omega_{n}-\mu_{\mathrm{I}}\right)^{2}-\mathbf{p}^{2}-m_{\pi_{3}}^{2}-\lambda \rho^{2}, \quad i G_{22}^{b}{ }^{-1}=\left(-\mathrm{i} \omega_{n}+\mu_{\mathrm{I}}\right)^{2}-\mathbf{p}^{2}-m_{\pi_{3}}^{2}-\lambda \rho^{2} \\
& \mathrm{i} G_{33}^{b}{ }^{-1}=\left(-\mathrm{i} \omega_{n}\right)^{2}-\mathbf{p}^{2}-m_{\pi_{3}}^{2}-2 \lambda v^{2}, \quad \mathrm{i} G_{44}^{b}{ }^{-1}=\left(-\mathrm{i} \omega_{n}\right)^{2}-\mathbf{p}^{2}-m_{\pi_{3}}^{2} .
\end{aligned}
$$

Here and in the following the $\pi_{3}$ mass is denoted by $m_{\pi_{3}}$ due the PMS relation $\left(m_{\pi_{3}}=M_{\pi_{3}}\right)$. In this way $M(T, \mu)$ is eliminated from (15) and the resummed $\pi_{3}$ mass is determined by the equation

$$
m_{\pi_{3}}^{2}(T, \mu)=m^{2}+\delta m^{2}+(\lambda+\delta \lambda)\left(v^{2}+\rho^{2}\right)+\Sigma_{\pi_{3}}\left(\omega=\mathbf{p}=0, m_{\pi_{3}}^{2}, T, \mu\right),
$$

where $\Sigma_{\pi_{3}}$ now depends on $m_{\pi_{3}}$ through the "resummed tree level" propagator matrix (16). This means that the above procedure makes the $\pi_{3}$ propagator $\left(G_{44}^{b}\right)$ selfconsistent at $p=0$, while the mixed sector of the boson propagator is partially resummed due to its $m_{\pi_{3}}$ dependence.

The self energy in (17) contains bosonic as well as fermionic loop integrals,

$$
\begin{aligned}
\Sigma_{\pi_{3}}\left(\omega=\mathbf{p}=0, m_{\pi_{3}}^{2}, T, \mu\right) & =\lambda+\lambda \sum_{\mathbf{p}} \operatorname{Tr}\left\{T^{b} G^{b}\left(\omega_{n}, \mathbf{p}, \mu_{\mathrm{I}}\right)\right\} \\
& +\lambda^{2} \sum_{\mathbf{p}} B_{i j}^{b} B_{k l}^{b}\left(G_{i k}^{b}\left(\omega_{n}, \mathbf{p}, \mu_{\mathrm{I}}\right) G_{j l}^{b}\left(\omega_{n}, \mathbf{p}, \mu_{\mathrm{I}}\right)+G_{i l}^{b}\left(\omega_{n}, \mathbf{p}, \mu_{\mathrm{I}}\right) G_{j k}^{b}\left(\omega_{n}, \mathbf{p}, \mu_{\mathrm{I}}\right)\right) \\
& +g_{F}^{2} \sum_{\mathbf{p}} G_{i l}^{f}\left(\omega_{n}, \mathbf{p}, \mu_{\mathrm{I}}, \mu_{\mathrm{B}}\right) B_{i j}^{f} G_{j k}^{f}\left(\omega_{n}, \mathbf{p}, \mu_{\mathrm{I}}, \mu_{\mathrm{B}}\right) B_{k l}^{f},
\end{aligned}
$$

where $T^{b}, B^{b, f}$ denotes the coupling matrices which arise from (9) and are listed in the appendix. It is worth to note that the divergences of the self energy are cancelled by the infinite perturbative counterterms if one replaces $m$ with $M(T, \mu)$ in (13). In this case, it seems that the counterterms are temperature and/or chemical potential dependent, however it can be proved order by order that all $T$ and/or $\mu$ dependent infinities are cancelled by higher order contributions (see for e.g. in [23] and [26]).

The scalar condensate $v$ is determined by the vanishing of the one-loop level one point function of $\sigma$ (EoS $\sigma$ at one-loop level),

$$
v\left(m^{2}+\delta m^{2}+(\lambda+\delta \lambda)\left(v^{2}+\rho^{2}\right)+\lambda \sum_{\mathbf{p}} \operatorname{Tr}\left\{H^{b} G^{b}\left(\omega_{n}, \mathbf{p}, \mu_{\mathrm{I}}\right)\right\}+g_{F} \sum_{\mathbf{p}} \operatorname{Tr}\left\{H^{f} G^{f}\left(\omega_{n}, \mathbf{p}, \mu_{\mathrm{I}}, \mu_{\mathrm{B}}\right)\right\}\right)=h,
$$

where $H^{b, f}$ can be found in the appendix. Comparing (18) and (19) one can recognize a Ward identity which connects the symmetry breaking external field with the propagator of $\pi_{3}$ at zero external momentum,

$$
v m_{\pi_{3}}^{2}=h .
$$

This relation is a consequence of the remaining $O(2)$ symmetry, which is an axial vector rotation around the third isospin axis from the point of view of the chiral symmetry. Moreover (20) guarantees the Goldstone theorem for this degree of freedom (neutral pion). 
The pion condensate is determined through the $\pi_{1}$ one point function, which is the EoS $\pi_{1}$ at one-loop level,

$$
\begin{aligned}
& \rho\left(m^{2}+\delta m^{2}+(\lambda+\delta \lambda)\left(v^{2}+\rho^{2}\right)-\mu_{\mathrm{I}}^{2}(1+\delta Z)+\lambda \sum_{\mathbf{p}} \operatorname{Tr}\left\{R^{b} G^{b}\left(\omega_{n}, \mathbf{p}, \mu_{\mathrm{I}}\right)\right\}\right. \\
& \left.+g_{F} \sum_{\mathbf{p}} \operatorname{Tr}\left\{R^{f} G^{f}\left(\omega_{n}, \mathbf{p}, \mu_{\mathrm{I}}, \mu_{\mathrm{B}}\right)\right\}\right)=0,
\end{aligned}
$$

where and $R^{b, f}$ can be found in the appendix. Here the infinite part of $\delta Z$ just cancels the $\mu_{\mathrm{I}}$ dependent divergence of the fermion loop integral.

The calculation of the loop integrals in (18) and (21) requires the diagonalization of the propagators and the corresponding transformation of the coupling matrices due to the non-diagonal matrix elements in (10) and (8). The diagonalization itself is a straightforward calculation, however the eigenvalues of the boson propagator (10) are non-rational functions of $\omega_{n}$. Thus performing the Matsubara sums is a very complicated task and it is beyond the scope of our paper. In order to avoid these difficulties the diagonalization was accomplished only for small $\rho$. As one shall see in the next section this method leads to ordinary Matsubara frequency dependence in the propagators. For that very reason we restricted ourselves to a Landau-type analysis of the pion condensation in a small vicinity of the phase boundary. Up to second order in $\rho$ the mass equation (17) can formally be written as

$$
m_{\pi_{3}}^{2}=m^{2}+\lambda v^{2}+t^{(0)}\left(m_{\pi_{3}}^{2}, v, T, \mu_{\mathrm{I}, \mathrm{B}}\right)+\left(\lambda+t^{(2)}\left(m_{\pi_{3}}^{2}, v, T, \mu_{\mathrm{I}, \mathrm{B}}\right)\right) \rho^{2},
$$

while (19) goes over into the simple form of (20) due to the Ward identity. At the same order in $\rho$ (21) can be rewritten as,

$$
\rho\left[\mu_{I}^{2}-m^{2}-\lambda v^{2}-r^{(0)}\left(m_{\pi_{3}}^{2}, v, T, \mu_{\mathrm{I}, \mathrm{B}}\right)-\left(\lambda+r^{(2)}\left(m_{\pi_{3}}^{2}, v, T, \mu_{\mathrm{I}, \mathrm{B}}\right)\right) \rho^{2}+\mathcal{O}\left(\rho^{4}\right)\right]=0
$$

By virtue of the above equation the pion condensate may have non-zero value only if the roots of the expression in the square bracket are real. Assuming that $\lambda+r^{(2)}>0$, Eq.(23) yields

$$
\rho=\sqrt{\frac{\mu_{I}^{2}-m^{2}-\lambda v^{2}-r^{(0)}\left(m_{\pi_{3}}^{2}, v, T, \mu_{\mathrm{I}, \mathrm{B}}\right)}{\lambda+r^{(2)}\left(m_{\pi_{3}}^{2}, v, T, \mu_{\mathrm{I}, \mathrm{B}}\right)}}
$$

if $\mu_{I}^{2}-m^{2}-\lambda v^{2}-r^{(0)}>0$. This means that in this case the transition is of second order supposing that the coefficient of the fourth order term in (23) is negative. Moreover if $\lambda+r^{(2)}<0$ and $\mu_{I}^{2}-m^{2}-\lambda v^{2}-r^{(0)}<0$ (keeping that the fourth order term is negative) it can be seen that the equation can have two nonzero roots, which suggests first order phase transition. For the calculation of the $t^{(0,2)}$ and $r^{(0,2)}$ coefficients in (22) and (24) diagonalization of the boson and fermion propagator up to order $\rho^{2}$ is needed, which will be presented in the next section.

\section{DIAGONALIZED PROPAGATORS FOR SMALL $\rho$}

In our approach, as was discussed previously, the next step is to determine the eigenvalues of the propagator matrices for small $\rho$ values, in other words to find the propagating eigenmodes with help of some suitable linear transformation of the original fields perturbatively in $\rho$. This step is not necessary if one would like to calculate the effective potential (see for e.g. [17]). In case of the bosonic propagator the transformation matrix up to $\mathcal{O}\left(\rho^{3}\right)$ is found to be,

$$
O_{B}=\left(\begin{array}{ccc}
1-|a|^{2} \rho^{2} & b(1-2 a v) \rho^{2} & -\sqrt{2} a \rho \\
b^{*}\left(1-2 a^{*} v\right) \rho^{2} & 1-|a|^{2} \rho^{2} & -\sqrt{2} a^{*} \rho \\
\sqrt{2} a \rho & \sqrt{2} a^{*} \rho & 1-2|a|^{2} \rho^{2}
\end{array}\right)+\mathcal{O}\left(\rho^{3}\right)
$$

where $a=a\left(\omega_{n}, \mu\right)=\lambda v /\left(\mu^{2}+2 \lambda v^{2}+2 \mathrm{i} \omega_{n} \mu\right)$ and $b=b\left(\omega_{n}, \mu\right)=\mathrm{i} \lambda /\left(4 \mu \omega_{n}\right)$. As it can be checked $O_{B}$ is not a unitary transformation and it is important to note that $O_{B}$ depends on the Matsubara frequency $\omega_{n}$. With this transformation $O_{B} \cdot\left(\mathrm{i} G_{\pi^{+}, \pi^{-}, \sigma}^{b}\right) \cdot O_{B}^{-1}=\operatorname{diag}\left(\mathrm{i} \tilde{G}_{\pi^{+}}^{-1}, \mathrm{i} \tilde{G}_{\pi^{-}}^{-1}, \mathrm{i} \tilde{G}_{\sigma}^{-1}\right)+\mathcal{O}\left(\rho^{3}\right)$, where the tilde reminds us that these propagators belong to the transformed (propagating) particles. It is worth to note that the new $\pi^{+}$and $\pi^{-}$particles are no longer charge conjugates of each other, which is a natural consequence of the presence of the pion condensate. After calculating the inverses perturbatively, the transformed $\pi^{+}, \pi^{-}$and $\sigma$ bosonic propagators are given by

$$
\mathrm{i} \tilde{G}_{\pi^{+}}=\frac{1}{\left(\omega_{n}+\mathrm{i} \mu_{\mathrm{I}}\right)^{2}+E_{\pi}^{2}}-\rho^{2} \frac{\lambda\left(2 \mu_{\mathrm{I}}^{2}+2 \lambda v^{2}-4 \mathrm{i} \mu_{\mathrm{I}} \omega_{n}\right)}{\left(\left(\omega_{n}+\mathrm{i} \mu_{\mathrm{I}}\right)^{2}+E_{\pi}^{2}\right)^{2}\left(\mu_{\mathrm{I}}^{2}+2 \lambda v^{2}-2 \mathrm{i} \mu_{\mathrm{I}} \omega_{n}\right)}+\mathcal{O}\left(\rho^{4}\right),
$$




$$
\begin{aligned}
\mathrm{i} \tilde{G}_{\pi^{-}} & =\frac{1}{\left(\omega_{n}-\mathrm{i} \mu_{\mathrm{I}}\right)^{2}+E_{\pi}^{2}}-\rho^{2} \frac{\lambda\left(2 \mu_{\mathrm{I}}^{2}+2 \lambda v^{2}+4 \mathrm{i} \mu_{\mathrm{I}} \omega_{n}\right)}{\left(\left(\omega_{n}-\mathrm{i} \mu_{\mathrm{I}}\right)^{2}+E_{\pi}^{2}\right)^{2}\left(\mu_{\mathrm{I}}^{2}+2 \lambda v^{2}+2 \mathrm{i} \mu_{\mathrm{I}} \omega_{n}\right)}+\mathcal{O}\left(\rho^{4}\right), \\
\mathrm{i} \tilde{G}_{\sigma} & =\frac{1}{\omega_{n}^{2}+E_{\sigma}^{2}}-\rho^{2} \frac{\lambda\left(\mu_{\mathrm{I}}^{2}+2 \lambda v^{2}\right)\left(\mu_{\mathrm{I}}^{2}+6 \lambda v^{2}+4 \mu_{\mathrm{I}}^{2} \omega_{n}^{2}\right)}{\left(\omega_{n}^{2}+E_{\sigma}^{2}\right)^{2}\left(\left(\mu_{\mathrm{I}}^{2}+2 \lambda v^{2}\right)^{2}+4 \mu_{\mathrm{I}}^{2} \omega_{n}^{2}\right)}+\mathcal{O}\left(\rho^{4}\right),
\end{aligned}
$$

while the $\pi_{3}$ propagator is

$$
\mathrm{i} G_{\pi_{3}}=\frac{1}{\omega_{n}^{2}+E_{\pi}^{2}}-\rho^{2} \frac{\lambda}{\left(\omega_{n}^{2}+E_{\pi}^{2}\right)^{2}}+\mathcal{O}\left(\rho^{4}\right) .
$$

In case of the fermionic inverse propagator matrix the diagonalization must be performed cautiously due to the presence of the non commuting Dirac matrices. The clearest approach is to solve the equation $O_{F}\left(\mathrm{i} G_{F}^{-1}\right) O_{F}^{-1}=\operatorname{diag}$ for $O_{F}$ directly. In this way $O_{F}$ is found to be

$$
O_{F}=\left(\begin{array}{cc}
1+\frac{g_{F}^{2}}{32 k_{0}^{2}} \rho^{2} & -\mathrm{i} \frac{g_{F}}{4 k_{0}} \gamma_{0} \gamma_{5} \rho \\
-\mathrm{i} \frac{g_{F}}{4 k_{0}} \gamma_{0} \gamma_{5} \rho & 1+\frac{g_{F}^{2}}{32 k_{0}^{2}} \rho^{2}
\end{array}\right),
$$

where $k_{0}=\left(-\mathrm{i} \omega_{n}+\frac{1}{3} \mu_{\mathrm{B}}\right) \gamma_{0}$ and the matrix is hermitian. After performing the inverse perturbatively the fermionic propagators are given by

$$
\mathrm{i} \tilde{G}_{u / d}=-\frac{1}{\not p_{u / d}-m_{f}}-\rho^{2} \frac{g_{F}^{2}}{8 k_{0}} \frac{1}{p_{u / d}-m_{f}} \gamma_{0} \frac{1}{p_{u / d}-m_{f}}
$$

where $p_{u / d}=\left(-\mathrm{i} \omega_{n}+\mu_{u / d}\right) \gamma_{0}-\gamma_{i} p_{i}$ and $\mu_{u / d}=\mu_{\mathrm{B}} / 3 \pm \mu_{\mathrm{I}} / 2$.

In the appendix it is shown that all integrandus appears in the one-loop equations (18), (19) and (21) can be written as traces over flavor space (in case of fermions traces also concern Dirac indices). In this way one can insert the bosonic/fermionic transformation matrices given in (25) and (30) under the traces and transform the propagators within into diagonal form, which will lead to transformation of the corresponding coupling matrices. Thus for instance the trace in A.4 can be written as

$$
\operatorname{Tr}\left\{B^{b^{\prime}} G^{b}\right\}=\operatorname{Tr}\left\{B^{b^{\prime}} O_{B}^{-1} O_{B} G^{b} O_{B}^{-1} O_{B}\right\}=\operatorname{Tr}\left\{O_{B} B^{b^{\prime}} O_{B}^{-1} \tilde{G}^{b}\right\}=\operatorname{Tr}\left\{\tilde{B}^{b} \tilde{G}^{b}\right\},
$$

where $\tilde{G}^{b}=\operatorname{diag}\left(\tilde{G}_{\pi^{+}}^{-1}, \tilde{G}_{\pi^{-}}^{-1}, \tilde{G}_{\sigma}^{-1}\right)$, and $\tilde{B}^{b}$ is the transformed coupling matrix, which depends on $\omega_{n}$ as was mentioned earlier. Similar expressions can be derived in case of fermions.

\section{THE PARAMETERIZATION}

Before calculating at finite temperature and non-zero chemical potentials one has to parameterize the model at $T=\mu_{\mathrm{I}, \mathrm{B}}=0$. We closely follow the method presented in [21, 22, 27]. Since $\rho=0$ at $\mu_{\mathrm{I}}=0$, there are five parameters, namely $m^{2}, \lambda, g_{F}, h$ and $v$, which can be fixed by setting four physical quantities - namely the pion, sigma, $\mathrm{u}$ and $\mathrm{d}$ quark masses plus the pion decay constant (through the PCAC relation) - to their physical values and by requiring the fulfillment of the (20) equation of state. At $T=\mu_{\mathrm{I}, \mathrm{B}}=0$ and $\rho=0$, the one-loop level $\pi_{3}$ inverse propagator can be written as

$$
\mathrm{i}\left(G_{\pi_{3}}^{1-\text { loop }}\right)^{-1}=(1-\delta Z) p^{2}-m^{2}-\delta m^{2}-(\lambda+\delta \lambda) v^{2}-\Sigma_{\pi_{3}}\left(p^{2}=0\right)-p^{2} \Sigma_{\pi_{3}}^{\prime}\left(p^{2}=0\right)-\tilde{\Sigma}\left(p^{2}\right),
$$

where $\Sigma_{\pi_{3}}^{\prime}=\partial \Sigma_{\pi_{3}} / \partial p^{2}$ and $\tilde{\Sigma}_{\pi_{3}} \sim \mathcal{O}\left(p^{4}\right)$. Fixing the physical $\pi_{3}$ mass $\left(M_{\pi}=138 \mathrm{MeV}\right)$ through the $\pi_{3}$ one-loop level propagator at $p^{2}=0$ and using the (17) mass resummation equation at $\rho=T=\mu_{\mathrm{I}, \mathrm{B}}=0$ one obtains

$$
M_{\pi}^{2}=m^{2}+\lambda v^{2}+3 \lambda\left(T_{0}^{b}\left(M_{\pi}, l_{b}\right)+T_{0}^{b}\left(m_{\sigma}, l_{b}\right)\right)+2 g_{F}^{2} N_{c} T_{0}^{f, \pi}\left(m_{f}, l_{f}\right),
$$

where $m_{\sigma}^{2}=M_{\pi}^{2}+2 \lambda v^{2}$ and $T_{0}^{b / f, \pi}$ denotes the bosonic/fermionic tadpole integrals at zero $T$ and $\mu_{\mathrm{I}, \mathrm{B}}$. In (34) the lack of bubble integrals is due to the fact that at $p^{2}=0$ they reduce to a linear combination of tadpoles. These tadpoles are finite on account of the $\delta m^{2}$ and $\delta \lambda$ counterterms (13). Note that new divergent terms do not appear in the tadpoles at finite temperature and/or non-zero chemical potentials, as it should be. The presence of $\delta Z$ in (33) 

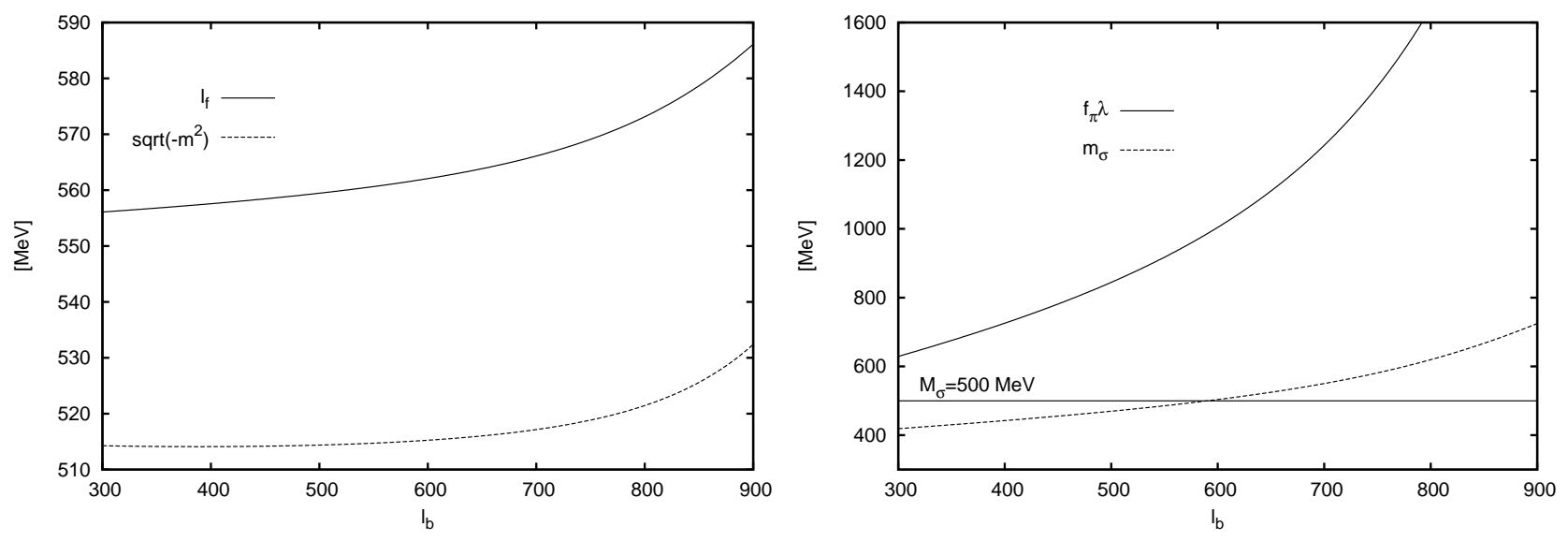

FIG. 1: Bosonic renormalization scale dependence of the fermion renormalization scale $l_{f}$, the mass parameter $m^{2}($ left panel) and of the coupling $\lambda$, the tree level $\sigma$ mass $m_{\sigma}$ (right panel).

is also important because this term renders the $p^{2}$ dependent part of the propagator finite. It involves also a finite renormalisation:

$$
\delta Z_{\pi_{3}}^{\mathrm{fin}}=\delta Z+\frac{\partial \Sigma_{\pi_{3}}}{\partial p^{2}}\left(p^{2}=0\right)=\frac{\lambda}{16 \pi^{2}} \frac{M_{\pi}^{4}-m_{\sigma}^{4}+M_{\pi}^{2} m_{\sigma}^{2} \log \left(\frac{m_{\sigma}^{2}}{M_{\pi}^{2}}\right)}{\left(m_{\sigma}^{2}-M_{\pi}^{2}\right)^{2}}+\frac{g_{F}^{2} N_{c}}{16 \pi^{2}} \log \left(\frac{\mathrm{e} m_{f}^{2}}{l_{f}^{2}}\right)
$$

and the one-loop level PCAC relation depends on $\delta Z_{\pi_{3}}^{\text {fin }}$ as follows

$$
v\left[\mathrm{i} G_{\pi_{3}}^{1-\mathrm{loop}}\left(p^{2}=0\right)\right]^{-1}=f_{\pi} M_{\pi}^{2}\left(1-\delta Z_{\pi_{3}}^{\mathrm{fin}} / 2\right)
$$

where $f_{\pi}=93 \mathrm{MeV}$ is the pion decay constant. Since $\delta Z_{\pi_{3}}^{\text {fin }}$ depends on the fermionic renormalization scale its actual value is tunable. Thus we required the vanishing of $\delta Z_{\pi_{3}}^{\text {fin }}$ to fix the fermionic renormalization scale. This requirement makes the PCAC relation simpler and thus the values of $v, g_{F}, h$ offer themselves immediately

$$
v=f_{\pi}, \quad g_{F}=2 \frac{m_{f}}{f_{\pi}}, \quad h=f_{\pi} M_{\pi}^{2},
$$

where $m_{f}=938 / 3 \mathrm{MeV}$ is the constituent $u, d$ quark mass. At this point $v, g_{F}$, and $h$ are known and $m^{2}$ can be expressed from (34).

The remaining unknown $\lambda$ parameter is determined by fixing the physical $\sigma$ mass at one-loop level and at zero external momentum, that is

$$
\begin{aligned}
M_{\sigma}^{2} & =m^{2}+\delta m+3(\lambda+\delta \lambda) v^{2}+\Sigma_{\sigma}\left(p^{2}=0\right) \\
& =m^{2}+3 \lambda v^{2}+3 \lambda\left(T_{0}^{b}\left(M_{\pi}, l_{b}\right)+T_{0}^{b}\left(m_{\sigma}, l_{b}\right)\right)+18 \lambda^{2} v^{2} B_{0}^{b}\left(m_{\sigma}, l_{b}\right)+6 \lambda^{2} v^{2} B_{0}^{b}\left(M_{\pi}, l_{b}\right)+6 g_{F}^{2} T_{0}^{f, \sigma}\left(m_{f}, l_{f}\right),
\end{aligned}
$$

where $B_{0}^{b}$ and $T_{0}^{f, \sigma}$ come from the temperature and chemical potential independent part of the bosonic/fermionic bubble diagram at zero external momentum and degenerate masses. It is worth to note that the same infinite counterterms render the above equation as well as the equation of $M_{\pi}$ finite.

We use $M_{\sigma}=500 \mathrm{MeV}$ for the physical $\sigma$ mass. This choice seems somewhat arbitrary, because the $\sigma$ meson is a broad resonance rather than a particle with well-defined mass (see [28] and references therein). Thus it would be more appropriate to identify the mass and width of the $\sigma$ meson through the pole of its spectral function [29]. However, we checked that varying $M_{\sigma}$ in the $400 \mathrm{MeV}-750 \mathrm{MeV}$ range produces just the same order of uncertainties in the thermodynamical results as the variation of the $l_{b}$ boson renormalization scale itself. Accordingly, it is enough to analyze the $l_{b}$ dependence of the different parameters, hence here and in the following we present our results which correspond to the choice $M_{\sigma}=500 \mathrm{MeV}$ at zero $T, \mu_{\mathrm{I}, \mathrm{B}}$. On the left panel of Fig. 1 the bosonic renormalization scale dependence of $m^{2}$ and $l_{f}$, while on the right panel the tree level $\sigma$ mass and the $\lambda$ parameter are shown. As one can see on the right panel, the tree level $\sigma$ mass equals its one-loop level value at $l_{b} \approx 600 \mathrm{MeV}$ thus the $\sigma$ mass becomes selfconsistent at this point (but only for zero $T, \mu_{\mathrm{I}, \mathrm{B}}$ !). In addition $m$ and $l_{f}$ moderately depend on the renormalization scale around this point (see left panel of Fig. 1). According to the above arguments we choose the following scale range: $l_{b} \in[400 \mathrm{MeV}, 800 \mathrm{MeV}]$ for the thermodynamical calculations. 

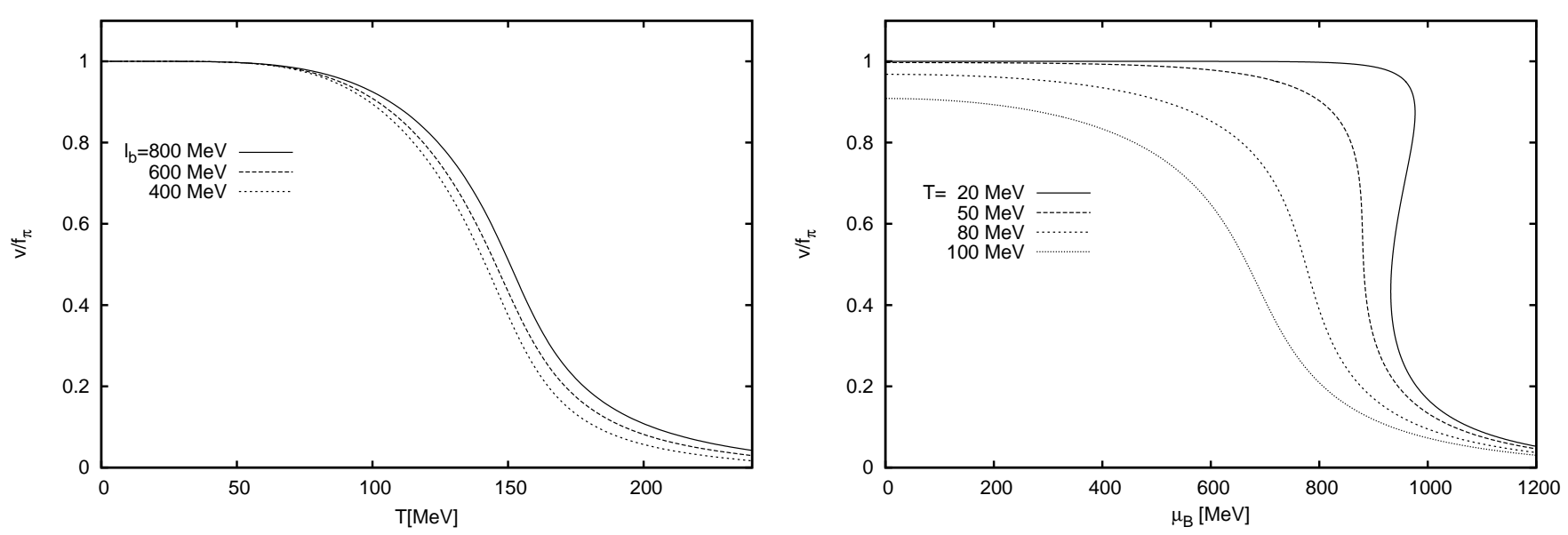

FIG. 2: Temperature and chemical potential dependence of the scalar condensate $v$. Left panel shows $v(T)$ at $\mu_{\mathrm{I}, \mathrm{B}}=0$ and $l_{b}=400,600,800 \mathrm{MeV}$. Right panel shows $v\left(\mu_{\mathrm{B}}\right)$ at $\mu_{\mathrm{I}}=0$ and $T=20,50,80,100 \mathrm{MeV}$.

\section{RESULTS AT LOWEST ORDER IN $\rho$}

As was derived in Sec. III the expectation value $\rho$ of the pion field is determined by (24) up to second order in $\rho$. From this equation it can be immediately seen that the second order boundary for the occurrence of the pion condensation in the $\mu_{\mathrm{I}}-\mu_{\mathrm{B}}-T$ space is determined by

$$
\mu_{I}^{2}-m^{2}-\lambda v^{2}-r^{(0)}\left(m_{\pi_{3}}^{2}, v, T, \mu_{\mathrm{I}, \mathrm{B}}\right)=0 .
$$

Moreover, the $\mu_{\mathrm{I}, \mathrm{B}}$ and $T$ dependence of $v$ and $m_{\pi_{3}}$ are determined by (20) and (22), which have to be solved at $\rho=0$. At $\rho=0$ (22) has the same form as (34) with the slight difference that now the tadpoles which appear therein have to be calculated at finite temperature and chemical potentials, while the form of (20) is unchanged. Using the explicit expression of $r^{(0)}$, Eq. (39) can be expressed as

$$
\mu_{I}^{2}-m_{\pi_{3}}^{2}\left(T, \mu_{\mathrm{I}}, \mu_{\mathrm{B}}\right)-R^{1-\mathrm{loop}}\left(T, \mu_{\mathrm{I}}, \mu_{\mathrm{B}}\right)=0,
$$

where $R^{1 \text {-loop }}$ is the remaining part of $r^{(0)}$ after subtracting from it $m_{\pi_{3}}^{2}$. It contains one-loop bosonic and fermionic contributions. From (40) it is obvious that at one-loop level the condensation does not start exactly at $\mu_{\mathrm{I}}=m_{\pi_{3}}$, as it is commonly expected, but it is shifted to some extent by $R^{1-\text { loop }}$. It's worth to note that the deviation $R^{1-\text { loop }}$ does not vanish identically even if the resummed pion mass is defined as the pole of the propagator.

First, we investigated the temperature and chemical potential dependence of the scalar condensate $v$ at $\mu_{\mathrm{I}}=0$ and different values of $l_{b}$ by solving (20) and (22), which can be seen in Fig. 2. On the left panel the temperature dependence of $v$ is shown at $l_{b}=400,600,800 \mathrm{MeV}$ and at zero chemical potentials. As it is expected, the scalar condensate shows a smooth crossover as the chiral symmetry is restored at a pseudocritical temperature $\left(T_{c}\right)$ around $150 \mathrm{MeV}$, which is in good agreement with the continuum limit lattice result found in [30]. Moreover, the $v(T)$ curve and consequently $T_{c}$ slightly depend on $l_{b}$. Hence, in the forthcoming we use the fixed scale $l_{b}=600 \mathrm{MeV}$. On the right panel the baryochemical potential dependence of $v$ can be seen at $l_{b}=600 \mathrm{MeV}$ scale, and at $T=20,50,80,100 \mathrm{MeV}$ temperatures. At small temperatures the transition is of first order, while for large temperatures it is of analytic crossover type, indicating the existence of a critical endpoint (CEP), where the transition changes from first order to crossover with increasing temperature (see e.g. in [21] and references therein). As one can see on Fig. 2 the temperature at the CEP is around $50 \mathrm{MeV}$, which is much lower than the lattice result presented in Ref. [31], however this is a common feature of effective models (for two/three flavors see e.g. Refs. [29], [21]). The critical/pseudocritical baryochemical potential values range from $\sim 600 \mathrm{MeV}$ to $\sim 1000 \mathrm{MeV}$ depending on the temperature.

Next, solving (20) and (22) for different $T, \mu_{\mathrm{I}}, \mu_{\mathrm{B}}$ values and tracking the fulfillment of the (40) condition we determined the second order critical surface of pion condensation, which can be seen on Fig. 3. As a function of the isospin chemical potential at fixed $\mu_{\mathrm{B}}$ the region of pion condensation starts very steeply at around $130 \mathrm{MeV}$. After that steep jump a plateau starts, which decreases slowly towards higher values of $\mu_{\mathrm{I}}$. Moreover, it can be seen that the region of pion condensation shrinks with increasing $\mu_{\mathrm{B}}$ and it even disappears at around $\mu_{\mathrm{B}}=830 \mathrm{MeV}$ (and $\mu_{\mathrm{I}}=131 \mathrm{MeV}$ ), a behavior which is in accordance with previous effective model studies (see e.g. Sec. VII.A of [17]). This is understandable physically, since at large $\mu_{\mathrm{B}}$ the condensate $\rho$ is basically determined by the difference of the 


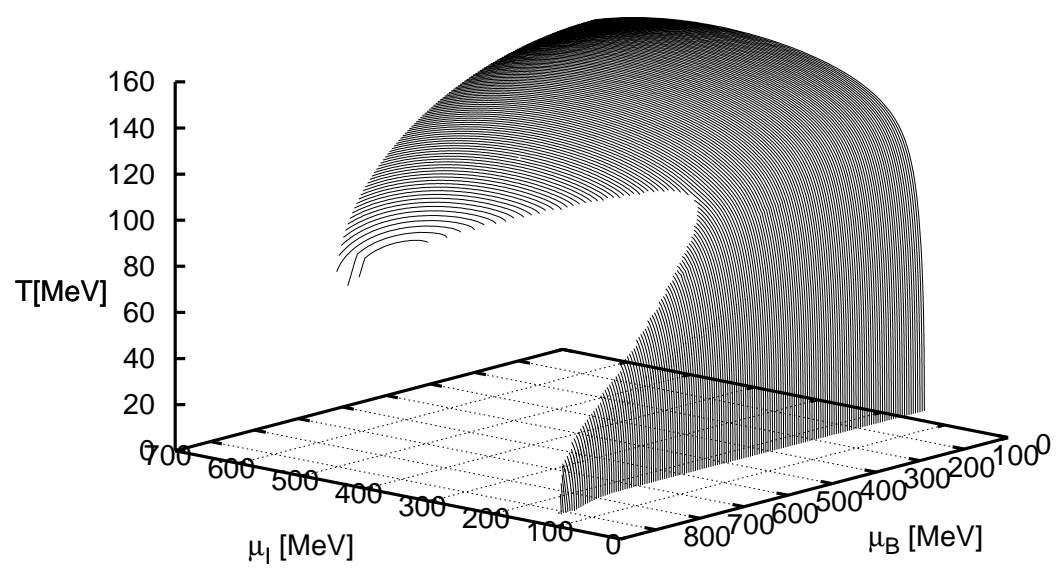

FIG. 3: Second order critical surface of the pion condensation in the $\mu_{\mathrm{I}}-\mu_{\mathrm{B}}-T$

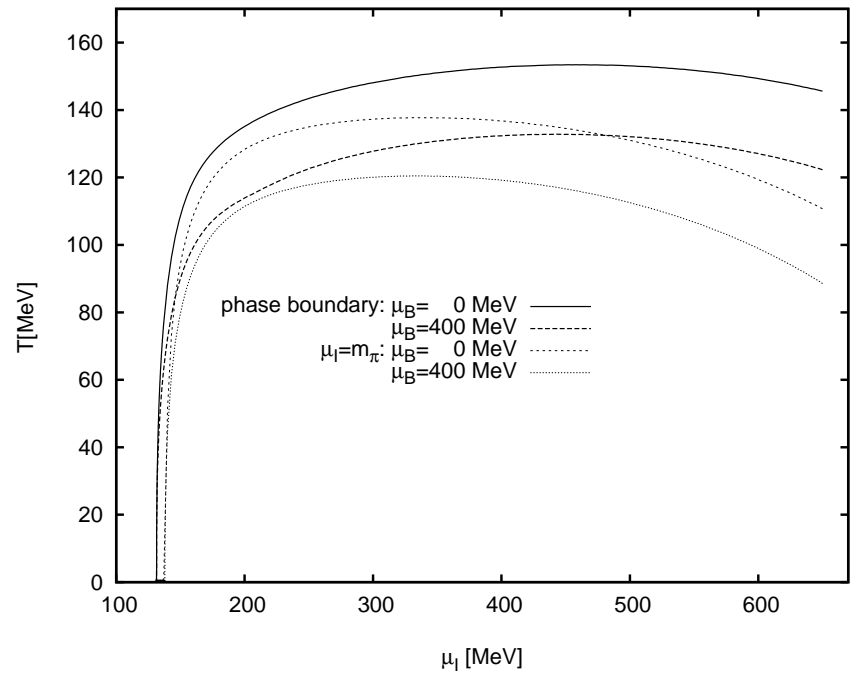

FIG. 4: Phase boundary of pion condensation and the $\mu_{\mathrm{I}}=m_{\pi_{3}}$ condition as function of the isospin chemical potential for different $\mu_{\mathrm{B}}$ values.

$u-d$ quark contributions in the EoS, and this difference is decreasing with increasing $\mu_{\mathrm{B}}$ at fixed $\mu_{\mathrm{I}}$, because the Fermi-Dirac factor in the contribution of $u$ depends on $\mu_{\mathrm{B}}+\mu_{\mathrm{I}}$, while in the contribution of $d$ on $\mu_{\mathrm{B}}-\mu_{\mathrm{I}}$. It is worth to observe that on the surface a gradually increasing missing part starts from about $\mu_{\mathrm{B}}=415 \mathrm{MeV}$ and $\mu_{\mathrm{I}}=221 \mathrm{MeV}$. In that region the $\mu_{I}^{2}-m^{2}-\lambda v^{2}-r^{(0)}$ combination (numerator of Eq. (24)) is negative, which means that the transition is not second order anymore. Strictly speaking if in this region there is a nonzero solution to $\rho$ this can happen only if $\lambda+r^{(2)}<0$ (denominator of Eq. (24) $)$ and in this case the transition is of first order.

To analyze the surface in detail, two sections taken at $\mu_{\mathrm{B}}=0 \mathrm{MeV}$ and $\mu_{\mathrm{B}}=400 \mathrm{MeV}$ are plotted in Fig. 4 together with the $\mu_{\mathrm{I}}=m_{\pi_{3}}$ curves. At both baryochemical potentials the condensation starts at around $\mu_{\mathrm{I}}=131 \mathrm{MeV}$, which is slightly below the $m_{\pi_{3}}=138 \mathrm{MeV}$ pion mass, this deviation is due to the corrections $R^{1-\mathrm{loop}}$ in (40). Moreover, the deviation widens as $\mu_{\mathrm{I}}$ increases. Another interesting feature is that at fixed high temperature as we increase the

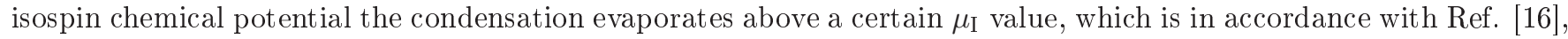
where this phenomenon was observed in case of two flavor PNJL model. In the region where the condensate already evaporated $(\rho=0)$ the chiral symmetry is almost totally restored $(v \approx 0)$.

Finally we calculated the one-loop pole masses of the charged pions on different temperatures as a function of the 


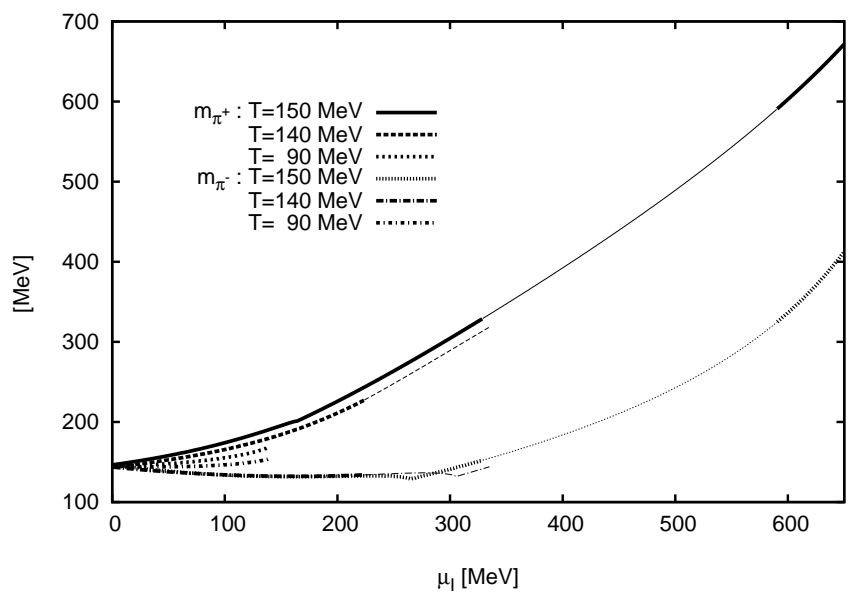

FIG. 5: $\mu_{\text {I }}$ dependence of the one-loop pole masses of charged pions at different temperatures $(T=90,140,150 \mathrm{MeV})$. The thin lines indicate the condensed phase for each temperature (where our calculation is not valid).

isospin chemical potential at $\rho=0$. For this we calculated the self energies of the charged pions, then we solved the following pole mass equations:

$$
\left(M_{\pi^{ \pm}}^{\text {pole }}\right)^{2}=\left(m_{\pi^{ \pm}}^{\text {tree }}\right)^{2}+\Sigma_{\pi^{ \pm}}\left(\omega=M_{\pi^{ \pm}}^{\text {pole }}, \mathbf{p}=0, T, \mu_{\mathrm{I}, \mathrm{B}}\right),
$$

where we have implicitly used the already known solutions $v\left(T, \mu_{\mathrm{I}, \mathrm{B}}\right)$ and $m_{\pi_{3}}\left(T, \mu_{\mathrm{I}, \mathrm{B}}\right)$. The one-loop masses are plotted on Fig. 5 . On the figure thick lines represent the sectors, where our calculation is valid, that is $\rho=0$. At the thin line parts of the curves the pions are condensed, thus we should go beyond the lowest approximation in $\rho$ to get correct results in that sectors. It is worth to note that when the condensation sets in none of the one-loop charged pion masses becomes zero as opposed to the masses defined through the dispersion relation (see e.g. [18]). Another interesting thing is that at larger values of $\mu_{\mathrm{I}}$ where the condensation has already evaporated and our calculation is valid (thick line parts) the charged pion masses are still different, however $\rho=0$. This difference is due to the fact that the isospin background acts differently on the charged pions (with opposite signs).

\section{CONCLUSIONS}

In this paper we studied the pion condensation in the framework of $S U(2)_{L} \times S U(2)_{R}$ constituent quark model with explicitly broken symmetry term in the presence of baryochemical potential. The model was parameterized at one-loop level and optimized perturbation theory was used for the resummation of the perturbative series. The one-loop equations were expanded in powers of $\rho$, and a Landau type analysis was performed for the phase boundary at lowest order in $\rho$. A simple condition for the boundary of the pion condensation was set up, and we argued that this condition gives a second order surface in the $\mu_{\mathrm{I}}-\mu_{\mathrm{B}}-T$ space. The temperature and renormalization scale dependence of the scalar condensate $v$ was investigated, and a mild renormalization scale dependence was found. At zero baryochemical potential the pseudocritical temperature is in accordance with results found on lattice [30]. Using the condition for the pion condensation the second order surface was determined. It was found that the surface starts steeply with increasing $\mu_{\mathrm{I}}$ at fixed $\mu_{\mathrm{B}}$ and towards large values of $\mu_{\mathrm{B}}$ the pion condensed region shrinks and even disappears at around $\mu_{\mathrm{B}}=830 \mathrm{MeV}$. However, at such a high energy one should take into account the effects of the strange quark. Investigating different sections of the surface it was showed that at one-loop level the pion condensation curve slightly differ from the $\mu_{\mathrm{I}}=m_{\pi_{3}}$ curve at small $\mu_{\mathrm{I}}$ and this deviation increases with increasing $\mu_{\mathrm{I}}$. We also studied the dependence of the charged one-loop pion masses on the isospin chemical potential. As a continuation of the present study the analysis can be extended to higher order in $\rho$, with which for instance the scaling properties around the surface, the dispersion relation at one-loop level and different phases of the condensed matter (BCS/LOFF) could be investigated. 


\section{Acknowledgment}

Work supported by the Hungarian Scientific Research Fund (OTKA) under contract numbers NI68228 and T046129. We thank A. Patkós and Zs. Szép for suggestions and careful reading of the manuscript.

\section{APPENDIX: COUPLINGS}

The coupling matrices appearing in (18), (19) and (21) can be obtained from the interaction term of the shifted fields in the Lagrangian

$$
\frac{\lambda}{4} \phi^{4}+\frac{g_{F}}{2} \bar{\psi} T_{\alpha} \phi_{\alpha} \psi \longrightarrow \frac{\lambda}{4}\left(\left(\pi_{1}+\rho\right)^{2}+\pi_{2}^{2}+\pi_{3}^{2}+(\sigma+v)^{2}\right)^{2}+\frac{g_{F}}{2} \bar{\psi}\left(\tau_{0}(\sigma+v)+\mathrm{i} \gamma_{5}\left(\tau_{1}\left(\pi_{1}+\rho\right)+\tau_{2} \pi_{2}+\tau_{3} \pi_{3}\right)\right) \psi .
$$

The four and three point couplings of (A.1) determine the coefficients of the tadpole and bubble terms of (18). Including the symmetry factors of the corresponding graphs these are:

$$
T^{b}=\left(\begin{array}{cccc}
1 & 0 & 0 & 0 \\
0 & 1 & 0 & 0 \\
0 & 0 & 1 & 0 \\
0 & 0 & 0 & 3
\end{array}\right), \quad B^{b}=\sqrt{2}\left(\begin{array}{cccc}
0 & 0 & 0 & \rho \\
0 & 0 & 0 & \rho \\
0 & 0 & 0 & \sqrt{2} v \\
\rho & \rho & \sqrt{2} v & 0
\end{array}\right), \quad B^{f}=\frac{\mathrm{i}}{2}\left(\begin{array}{cc}
-\gamma_{5} & 0 \\
0 & \gamma_{5}
\end{array}\right),
$$

where in case of bosons the same convention is used for the labeling of the matrix elements as in (10). The bosonic bubble contribution in (18) can be rewritten as

$$
\sum_{i, j, k, l} B_{i j}^{b} B_{k l}^{b}\left(G_{i l}^{b} G_{j k}^{b}+G_{j l}^{b} G_{i k}^{b}\right)=2 \operatorname{Tr}\left\{G^{b T} B^{b} G^{b} B^{b}\right\}
$$

and by virtue of the structure of $B^{b}$ and $G^{b}$ one can factor out the $\pi_{3}$ propagator from the above expression as follows

$$
\operatorname{Tr}\left\{G^{b T} B^{b} G^{b} B^{b}\right\}=G_{\pi_{3}}^{b} \operatorname{Tr}\left\{B^{b^{\prime}} G^{b}\right\},
$$

where

$$
B^{b^{\prime}}=2\left(\begin{array}{cccc}
\rho^{2} & \rho^{2} & \sqrt{2} v \rho & 0 \\
\rho^{2} & \rho^{2} & \sqrt{2} v \rho & 0 \\
\sqrt{2} v \rho & \sqrt{2} v \rho & 2 v^{2} & 0 \\
0 & 0 & 0 & 0
\end{array}\right) .
$$

The fermionic bubble contribution can be formulated as,

$$
\sum_{i, j, k, l} G_{i l}^{f} B_{i j}^{f} G_{j k}^{f} B_{k l}^{f}=\operatorname{Tr}\left\{G^{f T} B^{f} G^{f} B^{f}\right\}=\operatorname{Tr}\left\{B^{f} G^{f} B^{f} G^{f T}\right\},
$$

where the trace is over flavor as well as Dirac indices.

Moreover, in equations of states (19) and (21) the tadpole coefficients are also determined by the three point couplings of (A.1),

$$
R^{b}=\left(\begin{array}{cccc}
2 \rho & \rho & v / \sqrt{2} & 0 \\
\rho & 2 \rho & v / \sqrt{2} & 0 \\
v / \sqrt{2} & v / \sqrt{2} & \rho & 0 \\
0 & 0 & 0 & \rho
\end{array}\right), \quad R^{f}=\frac{1}{2}\left(\begin{array}{ll}
1 & 0 \\
0 & 1
\end{array}\right)
$$

and

$$
H^{b}=\left(\begin{array}{cccc}
v & 0 & \rho / \sqrt{2} & 0 \\
0 & v & \rho / \sqrt{2} & 0 \\
\rho / \sqrt{2} & \rho / \sqrt{2} & 3 v & 0 \\
0 & 0 & 0 & v
\end{array}\right), \quad H^{f}=\frac{\mathrm{i}}{2}\left(\begin{array}{cc}
0 & \gamma_{5} \\
\gamma_{5} & 0
\end{array}\right)
$$

[1] A. B. Migdal, Zh. Eksp. Teor. Fiz. 61, 2209 (1971). 
[2] A. B. Migdal, Phys. Rev. Lett. 31, 257 (1973).

[3] R. F. Sawyer, Phys. Rev. Lett. 29, 382 (1972).

[4] D. J. Scalapino, Phys. Rev. Lett. 29, 386 (1972).

[5] J. B. Kogut and D. K. Sinclair, Phys. Rev. D 66, 014508 (2002) arXiv:hep-lat/0201017.

[6] J. B. Kogut and D. K. Sinclair, Phys. Rev. D 66, 034505 (2002) arXiv:hep-lat/0202028.

[7] J. B. Kogut and D. K. Sinclair, Phys. Rev. D 70, 094501 (2004) arXiv:hep-lat/0407027.

[8] D. T. Son and M. A. Stephanov, Phys. Rev. Lett. 86, 592 (2001) arXiv:hep-ph/0005225].

[9] J. B. Kogut and D. Toublan, Phys. Rev. D 64, 034007 (2001) arXiv:hep-ph/0103271.

[10] A. Barducci, G. Pettini, L. Ravagli and R. Casalbuoni, Phys. Lett. B 564, 217 (2003) arXiv:hep-ph/0304019].

[11] B. Klein, D. Toublan and J. J. M. Verbaarschot, Phys. Rev. D 68, 014009 (2003) arXiv:hep-ph/0301143|.

[12] A. Barducci, R. Casalbuoni, G. Pettini and L. Ravagli, Phys. Rev. D 69, 096004 (2004) |arXiv:hep-ph/0402104].

[13] A. Barducci, R. Casalbuoni, G. Pettini and L. Ravagli, Phys. Rev. D 71, 016011 (2005) arXiv:hep-ph/0410250].

[14] D. Ebert and K. G. Klimenko, Eur. Phys. J. C 46, 771 (2006) arXiv:hep-ph/0510222].

[15] D. Ebert and K. G. Klimenko, J. Phys. G 32, 599 (2006) arXiv:hep-ph/0507007.

[16] Z. Zhang and Y. X. Liu, Phys. Rev. C 75, 064910 (2007) arXiv:hep-ph/0610221.

[17] L. y. He, M. Jin and P. f. Zhuang, Phys. Rev. D 71, 116001 (2005) |arXiv:hep-ph/0503272].

[18] J. O. Andersen, Phys. Rev. D 75, 065011 (2007) |arXiv:hep-ph/0609020|.

[19] H. Mao, N. Petropoulos and W. Q. Zhao, J. Phys. G 32, 2187 (2006) arXiv:hep-ph/0606241].

[20] J. O. Andersen and L. Kyllingstad, arXiv:hep-ph/0701033.

[21] P. Kovacs and Z. Szep, Phys. Rev. D 75, 025015 (2007) arXiv:hep-ph/0611208|.

[22] P. Kovacs and Z. Szep, Phys. Rev. D 77, 065016 (2008) arXiv:0710.1563 [hep-ph]].

[23] S. Chiku and T. Hatsuda, Phys. Rev. D 58, 076001 (1998) arXiv:hep-ph/9803226.

[24] M. Lévy, Nuovo Cim. A 52, 23 (1967).

[25] L. Dolan and R. Jackiw, Phys. Rev. D 9, 3320 (1974).

[26] A. Jakovac and Z. Szep, Phys. Rev. D 71, 105001 (2005) arXiv:hep-ph/0405226.

[27] T. Herpay and Z. Szep, Phys. Rev. D 74, 025008 (2006) [arXiv:hep-ph/0604086].

[28] W. M. Yao et al. [Particle Data Group], J. Phys. G 33, 1 (2006).

[29] A. Jakovac, A. Patkos, Z. Szep and P. Szepfalusy, Phys. Lett. B 582, 179 (2004) arXiv:hep-ph/0312088].

[30] Y. Aoki, Z. Fodor, S. D. Katz and K. K. Szabo, Phys. Lett. B 643, 46 (2006) arXiv:hep-lat/0609068.

[31] Z. Fodor and S. D. Katz, JHEP 0404, 050 (2004) arXiv:hep-lat/0402006. 